\title{
Diabetes and aspirin: beware of underpowered negative trials
}

\author{
Michael E Farkouh and Valentin Fuster
}

Diabetes mellitus afflicts over 20 million Americans and is responsible for a substantial proportion of cardiovascular events each year. In 1997 and on the heels of the US Physicians' Health Study where aspirin was shown to reduce rates of myocardial infarction in individuals with diabetes by $60 \%$, the American Diabetes Association strongly recommended the use of aspirin in primary prevention. This recommendation is currently under threat by two negative trials from the UK (Belch J et al. [2008] BMJ 337: a1840. doi: 10.1136/bmj.a1840) and Japan (Ogawa H et al. [2008] JAMA 300: 2134-2141).

There is no disputing the use of aspirin in patients with a prior cardiovascular or cerebrovascular event. In primary prevention, however, a meta-analysis by the Antithrombotic Trialists Collaboration (Antithrombotic Trialists' Collaboration [2002] BMJ 324: 71-86) reported a nonsignificant $7 \%$ reduction in cardiovascular events in the diabetic subpopulation and brought into question the widespread use of aspirin. Nevertheless, when the Adult Treatment Panel III lipid-lowering guidelines introduced the concept of diabetes as a coronary disease equivalent, the American Diabetes Association adopted a strong stance in support of aggressive treatment of diabetic individuals with any cardiovascular risk factors and included recommendations for use of aspirin.

Even when trials are conceived with the best of intentions, investigators are concerned with enrolling the required sample size in a timely fashion at any cost. There are a number of measures that can be adopted, including increasing the event rate by enriching the eligible population with higher-risk subjects. Another strategy, which is highly controversial, is to use a composite primary end point that includes several events. Often these events include softer end points, such as repeat revascularization, that are equally weighed with harder end points, such as cardiovascular death and myocardial infarction.

Two well-conducted clinical trials-the POPADAD (Prevention of Progression Of Arterial Disease and Diabetes) and the JPAD (Japanese
When an

important

public health

question is

on the line, we should be

prepared to

use adequately

powered trials

to attain a

reliable answer.

ME Farkouh is the

Director of the Mount

Sinai Heart Clinical

Trials Unit, Mount

Sinai Cardiovascular

Institute, NY, USA.

$V$ Fuster is the Editorin-Chief of Nature

Clinical Practice

Cardiovascular

Medicine, Director

of Mount Sinai

Heart, NY, USA, and

Scientific Director of

CNIC, Madrid, Spain.

Competing interests

The authors declared no

competing interests.

www.nature.com/clinicalpractice doi:10.1038/ncpcardio1433
Primary Prevention of Atherosclerosis With Aspirin for Diabetes) studies-enrolled diabetic patients who had no cardiovascular disease and found that low-dose aspirin ( $81-100 \mathrm{mg} /$ day) did not reduce a cluster of cardiovascular events. Although POPADAD used an abnormal anklebrachial index to enrich the study population, the trial was grossly underpowered. In the larger JPAD trial, the trend towards a $20 \%$ reduction in the primary outcome measure failed to meet statistical significance even though the study included the development of peripheral arterial disease and stable angina as primary events. Indeed, the reduction would have been significant if only harder end points, such as cardiovascular death and myocardial infarction, had been considered. These two trials underscore the paradoxical dilution effect of underpowered negative trials that have used softer endpoints to enhance statistical power.

The problem of low power also arises when trialists make unrealistic projections of event rates. In the JPAD trial, the investigators predicted 52 primary cardiovascular events per 1,000 person-years but the actual event rate was only 17 events per 1,000 person-years. This miscalculation occurs frequently in the cardiovascular arena because the rapid advent of new and improved therapies is driving event rates lower over the course of these trials.

When an important public health question is on the line, we should be prepared to use adequately powered trials to attain a reliable answer. These two trials of aspirin in patients with diabetes mellitus do not tell us if aspirin should be used in such populations. Accordingly, we should not abandon the guidelines for use of low-dose aspirin in the primary prevention of cardiovascular events in diabetic patients. Instead, the cardiovascular community should carefully review these two aspirin trials, beginning with an honest assessment of their limitations. The POPADAD and JPAD trials have not ruled out small but clinically important benefits of treatment with aspirin. We cannot afford to get it wrong. 